\title{
Estrategia de eficiencia en el consumo de energía eléctrica y mitigación en la estructura productiva de México
}

\author{
Efficiency strategy in electricity consumption and mitigation \\ in the productive structure of Mexico
}

\section{Jaime Mario Edmundo Vaca Serrano*, Antonio Kido Cruz}

Universidad Michoacana de San Nicolás de Hidalgo, México

Recibido el 1 de marzo de 2019; aceptado el 7 de mayo de 2020

Disponible en Internet el: 10 de junio de 2020

\section{Resumen}

En el presente estudio se realiza un análisis multisectorial del consumo de energía eléctrica en México, de la intensidad y eficiencia en dicho consumo, así como de la emisión de contaminantes por tipo de tecnología de generación de electricidad; con la finalidad de proponer una estrategia de mejora. Los resultados indican la existencia de 19 subsectores claves en el uso de la energía eléctrica. La mayor intensidad en el consumo de electricidad está en los subsectores de suministro de agua y gas por ductos, fabricación de productos a base de materiales no metálicos y fabricación de insumos textiles y acabados de textiles. La mayor emisión de $\mathrm{CO}_{2}$ corresponde a tecnologías de ciclo combinado, termoeléctrica convencional y carboeléctrica con una emisión de 122.7 Mt de $\mathrm{CO}_{2}$; las energías limpias emiten $2.7 \mathrm{Mt}$ de $\mathrm{CO}_{2}$. Con base en estos resultados se propone una estrategia de eficiencia en el consumo de electricidad y mitigación para el aparato productivo nacional.

Código JEL: E2, L94, Q43, Q51, Q54

Palabras clave: Eficiencia; Análisis multisectorial; Energía eléctrica; Mitigación

\footnotetext{
*Autor para correspondencia

Correo electrónico: jaimevaka@ hotmail.com (J. M. E. Vaca Serrano).

La revisión por pares es responsabilidad de la Universidad Nacional Autónoma de México. 


\begin{abstract}
This study conducts a multisectoral analysis of the consumption of electricity in Mexico, the intensity and efficiency of electricity consumption, as well as the emission of pollutants by type of electricity generation technology; in order to propose an improvement strategy. The results indicate the existence of 19 key subsectors in the use of electricity power. The greatest intensity in electricity consumption is in the subsectors of water and gas supply by pipelines, manufacturing of products based on non-metallic materials and manufacture of textile input and textiles finishes. The highest $\mathrm{CO}_{2}$ emission corresponds to combine cycle generation, conventional thermoelectric and coal-fired technologies with an emission of 122. Mt of $\mathrm{CO}_{2}$; clean energies emit 2.7 Mt of $\mathrm{CO}_{2}$. Based on these results, a strategy of efficiency in electricity consumption an mitigation for the national production apparatus is proposed.
\end{abstract}

JEL Code: E2, L94, Q43, Q51, Q54

Keywords: Efficiency; Multisectoral analysis; Electrical power; Mitigation

\title{
Introducción
}

De acuerdo con la Organización de Naciones Unidas (ONU, 2019), en la actualidad el cambio climático es un tema de interés general y representa uno de los desafíos más grandes al que nos enfrentamos. Los efectos de estos cambios son de magnitud global y de una proporción nunca antes vista, por lo que es necesario proponer estrategias que permitan mejorar la mitigación. El cambio climático es innegable, de acuerdo con la Universidad de Cambridge y el Consejo Mundial de Energía (2014, pp. 2-5). Muy probablemente el motivo preponderante sea originado por las actividades del ser humano, tales como las emisiones de dióxido de carbono.

La generación de electricidad es una de las principales fuentes emisoras de Gases de Efecto Invernadero (GEI), y también uno de los principales motores para el desarrollo del país, por lo que se han realizado numerosos estudios con objeto de determinar medidas de eficiencia energética con gran impacto en la reducción de emisiones, a fin de ponerlas en práctica sin afectar el crecimiento económico en México (INECC y PNUD, 2012, p. 22). Realizar un análisis multisectorial del consumo de energía eléctrica en México, así como de la intensidad, eficiencia y la emisión de contaminantes por tecnología de generación de electricidad, permitirá proponer una estrategia de mejora.

\section{Revisión de la literatura}

El análisis de Insumo-Producto de energía detalla el consumo y uso de energía necesaria para producir bienes y servicios en cada uno de los sectores de la estructura productiva de 
una región o país. Existen tres modelos elementales de este tipo de análisis (Guevara et al., 2018, p. 7): el modelo de unidad híbrida de Bullard y Herendeen (1975), el de coeficiente de efecto directo basado en la intensidad de energía directa de todos los sectores (Miller y Blair, 2009), y el modelo multifactor que explica los flujos de energía de acuerdo con los procesos de conversión de energía en la economía (Guevara y Domingos, 2017).

Ruíz Nápoles ha realizado varios estudios referentes al crecimiento bajo en carbono y adopción de tecnologías para la mitigación en Argentina y Brasil (Ruiz, 2014a), así como ha propuesto políticas de mitigación del cambio climático en México (2014b), concluyendo que las ramas del sector energético son las que más contaminantes emiten e impulsan de manera indirecta, contribuyendo a las emisiones de gases de efecto invernadero.

Cardenete y Fuentes-Saguar (2014) analizaron el sector energético de Andalucía y su importancia desde el punto de vista del consumo final de energía, identificando, mediante el uso de matriz de contabilidad social, qué sectores son los de mayor responsabilidad en la emisión de contaminantes y de mayor costo en términos de emisión de $\mathrm{CO}_{2}$.

Gianialli y Canziani (2014) determinaron que el desarrollo bajo en carbono (LCDS) en Perú expone una gran mejora, tanto en beneficios económicos, sociales y ambientales como en la incorporación de financiamiento y aplicación de tecnologías disponibles internacionalmente. Betancourt (2015) determina los efectos de la aplicación de un impuesto ambiental al $\mathrm{CO}_{2}$ en México enfatizando que la implementación de políticas fiscales e instrumentos económicos con enfoque climático impulsará el desarrollo económico bajo en emisiones y elevará la competitividad. Loizou y Chatzitheodoridis (2015) analizaron la dinámica del sector energético griego, determinando como los sectores energéticos coadyuvan de manera fundamental en la economía nacional, identificando su encadenamiento hacia atrás y hacia adelante con los otros sectores de la economía.

Livas-García (2015) mediante el concepto de requerimientos energéticos de energía a través de la metodología insumo-producto y el análisis de cambio estructural, determinó la dependencia del sistema energético mexicano de los hidrocarburos. Guevara, Rodríguez y Domingos (2015) introdujeron un nuevo modelo de insumo-producto de energía, denominado modelo final, que describe los flujos de energía de acuerdo con los procesos de conversión de energía y los niveles de uso de energía en la economía; por otra parte Guevara, Souza y Domingos (2016) presentaron información sobre las transiciones de energía en México a partir del análisis de la exergía útil 1971-2009, la exergía es una medida de energía disponible, y se denomina exergía útil al mínimo valor necesario de trabajo requerido para producir un uso final dado, lo cual permite medir la cantidad efectiva de exergía que se entrega a una función final.

Patiño (2016) analiza la estructura productiva, eficiencia energética y emisiones de $\mathrm{CO}_{2}$ en Colombia, identificando y midiendo los elementos fundamentales que contribuyen a estas emisiones y su conexión con el consumo de energía. La Secretaría de Energía (SENER, 2016) 
publicó la determinación de la línea base de consumo energético y potenciales de eficiencia energética sectoriales en México mediante el análisis de los sectores industria, transporte, residencial, comercial, usos no energéticos y agropecuario.

Liu, Chen, Tian, Zheng y Li (2016) concluyen que la promoción de energías de combustibles no fósiles ofrecerá una contribución creciente a una transición baja en carbono en el mediano y largo plazo en China. Arreola-Marroquín y Ríos (2017) determinan que la tasa de crecimiento de la producción y el consumo de energía se ven afectados negativamente por la tasa de crecimiento del precio de la energía. Consecuentemente, las políticas económicas deben centrarse mucho más directamente en el aumento de la eficiencia energética y la innovación.

Guevara, Córdova, García y Bouchain (2017) analizan el estado y la evolución de la energía en México antes de la reforma energética de 2014, identifican la presencia de tres elementos decisivos en el cambio del uso y consumo de energía primaria, así como determinan que el sector energético presenta un estado deficiente en cuanto a su estructura y operación. Mediante la aplicación del denominado modelo multifactor de energía, Guevara et al. (2018) determina las relaciones de energía y emisiones de $\mathrm{CO}_{2}$ en el bloque (TLCAN), de manera que los resultados muestran que el TLCAN no cuenta con un sistema integrado conjunto de energía para los tres países participantes, aunque ha ayudado a reducir las emisiones de $\mathrm{CO}_{2}$ relacionadas con la energía.

Esta revisión de la literatura mostró diferentes estudios sobre el consumo de energía, la eficiencia energética, las emisiones de $\mathrm{CO}_{2}$ y la mitigación en la estructura productiva, la mayoría realizados mediante la aplicación del análisis insumo-producto, y referidas al uso y consumo de energía considerando en conjunto los hidrocarburos (petróleo, gas y derivados) y la electricidad; por lo que no existe un análisis exclusivo del sector eléctrico. En virtud de lo anterior, el objetivo de este trabajo es realizar un análisis multisectorial del consumo de energía eléctrica, de la intensidad y eficiencia, a fin de determinar los subsectores claves en el consumo de energía eléctrica a partir de la base de datos de la matriz Insumo-Producto publicada en octubre de 2017 por el Instituto Nacional de Estadística y Geografía (INEGI) a precios básicos de 2013; así como de la emisión de contaminantes por tecnología de generación de electricidad con datos a 2017, con objeto de proponer una estrategia de mejora.

\section{Emisiones de CO2 por tipo de fuente y tecnología de generación}

Diferentes fuentes y tecnologías están presentes en la generación de electricidad. Actualmente existen varias opciones con particularidades muy diferentes, tal que la principal clasificación en México distingue energías convencionales y energías limpias. El potencial de recursos renovables y energía limpia en México es inmenso; la generación de electricidad cuenta con una diversidad significativa de tecnología para su explotación. Las centrales hidroeléctricas 
son las de mayor aportación en la capacidad instalada, seguidas de las centrales geotermoeléctricas, eólicas, y solar fotovoltaica. Estas últimas dos fuentes han tenido el mayor crecimiento en los últimos años, (CEPAL, 2018, p. 83). El ritmo de crecimiento más acelerado lo presentó la eólica que creció en capacidad instalada de 3.735 MW en 2016 a 5.077 MW en 2018; por su parte la solar fotovoltaica creció en capacidad instalada de 389 MW en 2016 a 1646.55 MW en junio de 2018.

Puesto que el sector eléctrico es uno de los principales responsables de la emisión de Gases de Efecto Invernadero (GEI), la disminución en su consumo es trascendental para el logro de los objetivos establecidos. Existen dos formas de lograr la disminución en el consumo de energía eléctrica: reduciendo las actividades consumidoras en términos absolutos, o incrementado la eficiencia en el uso de la energía eléctrica en las diversas actividades. Se considera el segundo caso más factible dado que no implica una disminución en la actividad económica, pese a no obtener disminuciones absolutas (Mendiluce y Linares, 2011, pp. 1-3).

De acuerdo con el Inventario Nacional de Emisiones de Gases y Compuestos de Efecto Invernadero, del Instituto Nacional de Ecología y Cambio Climático (INECC), las tecnologías convencionales para la generación de electricidad contribuyen con el $18 \%$ de las emisiones totales de Gases de Efecto Invernadero (GEI) a nivel nacional (es la segunda actividad del país con mayor impacto al medio ambiente, después del transporte), con un volumen equivalente a 125 mil millones de toneladas de CO2 (SENER, 2018, p. 25).

La Tabla 1 presenta las emisiones emitidas por fuente de generación, el promedio de emisiones por MWh de generación para las tecnologías convencionales y el tipo de tecnología existente en el año 2017.

Es conveniente indicar la importancia de la incorporación de Certificados de Energía Limpia (CEL) en la estructura eléctrica de México, los cuales son títulos emitidos por la Comisión Reguladora de Energía (CRE) con la finalidad de autentificar una cantidad medida de generación de electricidad a través de energía limpias y que asiste en el cumplimiento de las obligaciones asociadas al consumo de los centros de carga (CRE, 2017). El marco regulatorio en la materia proporciona a los inversionistas ciertos beneficios, entre los cuales destacan incentivos fiscales y certificados de promoción como son los CEL's, que pueden comercializarse en el mercado de acuerdo con la oferta y demanda de los mismos. 
Tabla 1

Emisiones de CO2 por tipo de Tecnología y fuente de generación 2017

\begin{tabular}{|c|c|c|c|c|}
\hline Tipo de tecnología & Fuente de generación & $\begin{array}{l}\text { Promedio de emisiones } \\
\text { por } \mathrm{MWh}\left(\mathrm{kg} \mathrm{de} \mathrm{CO}_{2}\right)\end{array}$ & $\begin{array}{l}\text { Centrales en } \\
\text { operación }\end{array}$ & $\begin{array}{c}\text { Emisiones emitidas } \\
\left.\text { (Mt de } \mathrm{CO}_{2}\right)\end{array}$ \\
\hline Convencional & & & 526 & 122.7 \\
\hline Ciclo combinado & Gas natural & 346 & 83 & 57.2 \\
\hline $\begin{array}{l}\text { Termoeléctrica } \\
\text { convencional }\end{array}$ & Derivados del petróleo & 680 & 59 & 29.1 \\
\hline Carboeléctrica & Carbón & 773 & 3 & 23.6 \\
\hline Turbogás & Gas natural y diésel & $\begin{array}{l}509 \\
660\end{array}$ & 131 & 7.3 \\
\hline $\begin{array}{l}\text { Combustión in- } \\
\text { terna }\end{array}$ & $\begin{array}{l}\text { Diésel, combustóleo o } \\
\text { mezcla de ambos }\end{array}$ & 688 & 248 & 2.8 \\
\hline Lecho fluidizado & Coque de petróleo & & 2 & 2.7 \\
\hline Limpia & & & 270 & 2.7 \\
\hline Renovable & & & 239 & 0.3 \\
\hline Hidroeléctrica & Agua & & 86 & 0.0 \\
\hline Eólica & Viento & & 45 & 0.0 \\
\hline Geotérmica & Vapor endógeno & & 8 & 0.0 \\
\hline Solar & Sol & & 23 & 0.0 \\
\hline Bioenergía & Biomasa y residuos & & 77 & 0.3 \\
\hline Otras & & & 31 & 2.4 \\
\hline Nucleoeléctrica & Uranio o plutonio & & 1 & 0 \\
\hline $\begin{array}{l}\text { Cogeneración efi- } \\
\text { ciente }\end{array}$ & $\begin{array}{c}\text { Energía primaria del } \\
\text { proceso }\end{array}$ & & 30 & 2.4 \\
\hline Total & & & 796 & 125.4 \\
\hline
\end{tabular}

Fuente: PRODESEN 2018-2032 (SENER, 2018, p. 141)

\section{Modelo Insumo-Producto de energía aplicado a energía eléctrica}

El consumo final de energía eléctrica está en función de los consumos sectoriales de la estructura productiva de la economía, del contenido energético, de la producción de cada sector y de la generación de capital; por lo que es muy valioso determinar el enlace del comportamiento económico sectorial con el consumo y demanda de energía eléctrica (Alcántara y Padilla, 2002, 
pp. 2-3). Un análisis multisectorial del consumo de energía eléctrica facilita la aplicación de indicadores de eficiencia energética, los cuales son un instrumento valioso para describir a detalle como el uso de la energía eléctrica es afectada por ciertos factores en los diferentes sectores de la economía, a la vez que facilitan identificar procesos en los cuales existen áreas de oportunidad en la mejora de la eficiencia energética y el alcance de ahorro por sector. A partir del conocido modelo de Leontief y siguiendo de forma similar para la energía eléctrica el análisis realizado por Hartner (2013, p. 109), a partir de una matriz de insumo-producto, podemos expresar la intensidad del consumo de electricidad como:

$$
C E E_{j}^{t}=\frac{E E F_{j}^{t}}{X_{j}}
$$

Dónde $\boldsymbol{C} \boldsymbol{E} \boldsymbol{E}_{\boldsymbol{j}}^{\boldsymbol{t}}$ es el consumo de energía eléctrica del suministrador $\boldsymbol{t}$ para el sector $\boldsymbol{j}$ en (kW/pesos), $\boldsymbol{E} \boldsymbol{E} \boldsymbol{F}_{\boldsymbol{j}}^{\boldsymbol{t}}$ es el uso final de energía eléctrica del suministrador $\boldsymbol{t}$ para el sector $\boldsymbol{j}$ en (kW/año) y $\boldsymbol{X}_{\boldsymbol{j}}$ es la producción total del sector $\boldsymbol{j}$ en (Pesos/año). Por lo que, considerando la matriz inversa de Leontief $(\boldsymbol{I}-\boldsymbol{A})^{-\mathbf{1}}$ la cual es conocida como la matriz de requerimientos totales $(\boldsymbol{R})$ y se puede expresar como de la siguiente manera:

$$
R=(I-A)^{-1}=\left[\begin{array}{cccc}
r_{11} & r_{12} & \ldots & r_{1 n} \\
r_{21} & r_{22} & \ldots & r_{2 n} \\
\ldots & \ldots & \ldots & \ldots \\
r_{n 1} & r_{n 2} & \ldots & r_{n n}
\end{array}\right]
$$

Al multiplicar esta matriz $(\boldsymbol{R})$ por una matriz diagonal $(\hat{\boldsymbol{e}})$ que expresa el consumo de energía eléctrica de las industrias en la diagonal (la cual está dada por la división del renglón de consumo de energía eléctrica entre la producción total), obtenemos una matriz que contiene los requisitos totales (producción total) de energía eléctrica para la producción de una unidad para la demanda final. Las columnas contienen la entrada de energía eléctrica a lo largo de la cadena de suministro de un producto y la suma de las columnas indica las entradas totales de energía eléctrica para producir una unidad de un producto industrial para la demanda final.

$$
R E E=\hat{e} \cdot(I-A)^{-1}=\left[\begin{array}{cccc}
r_{11} e_{1} & r_{12} e_{1} & \ldots & r_{1 n} e_{1} \\
r_{21} e_{2} & r_{22} e_{2} & \ldots & r_{2 n} e_{2} \\
\ldots & \ldots & \ldots & \ldots \\
r_{n 1} e_{n} & r_{n 2} e_{n} & \ldots & r_{n n} e_{n}
\end{array}\right]
$$

Donde $\boldsymbol{R E} \boldsymbol{E}$ son los requerimientos totales de energía eléctrica, ( $\widehat{\boldsymbol{e}}$ ) es la matriz diagonal con el consumo de energía eléctrica de las industrias en la diagonal y $(\boldsymbol{I}-\boldsymbol{A})^{\mathbf{- 1}}$ es la matriz inversa de Leontief. Junto con la ecuación original para la producción total, obtenemos el requerimiento total de energía eléctrica para la economía en función de la matriz de demanda 
final $(\boldsymbol{y})$.

$$
R E E=\widehat{e} \cdot(I-A)^{-1} \cdot y
$$

Al multiplicar la demanda como una matriz diagonal, se obtiene un vector de los requerimientos totales de energía eléctrica para el consumo de productos producidos por todas las industrias dentro de un año.

$$
R E E_{p g}=\widehat{e} \cdot(I-A)^{-1} \cdot \hat{y}
$$

En la aplicación de la metodología se ve con mayor claridad y detalle la aplicación del modelo insumo-producto de energía aplicado a la energía eléctrica.

\section{Índices de Rasmussen}

Dentro de los métodos existentes para identificar los sectores clave de una economía, esencialmente los que analizan el peso de cada sector de generar efectos de arrastre, tanto hacia adelante como hacia atrás, sin importar su tamaño, se cuenta con los denominados Índices de Rasmussen, los cuales tienen la característica de contar con una aplicación extensa, dado que proporcionan un primer acercamiento muy apropiado en el análisis de la estructura de una economía real (Núñez y Romero, 2016, p. 12).

La capacidad de originar impactos de arrastre hacia atrás corresponde a la magnitud que un incremento presentado en el sector origina en los demás sectores; y la capacidad de originar impactos hacia adelante se identifica como la magnitud en que el sistema económico pesa sobre la industria, es decir la medida en que la industria es afectada por un aumento en el sistema económico (Parra y Pino, 2003, p. 19). La forma general del modelo de Leontief está dada por la siguiente ecuación:

$$
x=L y
$$

En donde $\boldsymbol{x}$ representa el vector de producto total para cada sector de la economía, $\boldsymbol{L}=(\boldsymbol{I}-\boldsymbol{A})^{-\boldsymbol{I}}$ es la matriz de multiplicadores (inversa de Leontief) y $\boldsymbol{y}$ es el vector de demandas finales. Cada valor en cada sector (columna) de $\boldsymbol{L}$ se explica cómo el efecto de un incremento unitario exógeno correspondiente a ese sector, sobre el producto de cada sector productivo, de manera tal que la suma de la columna equivale al efecto multiplicador total. 
Es decir que, los índices de Rasmussen relacionan comparativamente el efecto en cada sector con el efecto medio de todos los sectores, tanto por arrastre (columna) como por dispersión (fila); por lo que si el impacto de un sector es superior a la media, su índice será mayor que uno (Núñez y Romero, 2016, p. 12). Dicho de otro modo, por columna el índice de arrastre o de impacto, se define como:

$$
\text { Índice de Arrastre }=U_{j}=\frac{\bar{m}_{j}}{\frac{1}{n} \sum m_{j}}
$$

En donde $\boldsymbol{i} \boldsymbol{j}=\mathbf{1}, \ldots, \boldsymbol{n}, \boldsymbol{n}$ es el número de sectores productivos, y $\overline{\boldsymbol{m}}_{\boldsymbol{j}}$ es el impacto medio del sector o cuenta $\boldsymbol{j}$ sobre los demás sectores. Del mismo modo, por fila el índice de dispersión se define como:

$$
\text { Índice de Dispersión }=U_{i}=\frac{\bar{m}_{i}}{\frac{1}{n} \sum m_{i}}
$$

En donde: $\boldsymbol{i} \boldsymbol{j}=\mathbf{1}, \ldots, \boldsymbol{n}, \boldsymbol{n}$ son el número de sectores productivos; y $\overline{\boldsymbol{m}}_{\boldsymbol{i}}$ es el impacto medio del sector o cuenta $\boldsymbol{i}$ sobre los demás sectores.

La clasificación de los sectores se establece de acuerdo con los valores obtenidos para los índices de arrastre (suma total de cada columna) y de dispersión (suma total de cada fila), con lo cual al comparar con la media se clasifica cada sector de la economía de un país. Los criterios correspondientes son los presentados en la Tabla 2.

\section{Tabla 2}

\begin{tabular}{|c|c|}
\hline Tipo de Sector & Interpretación \\
\hline Sectores Clave: & $\begin{array}{l}\text { Son los sectores de mayor integración con los otros sectores } \\
\text { del conjunto económico. }\end{array}$ \\
\hline \multicolumn{2}{|l|}{ Ambos índices $>1$. } \\
\hline Sectores Impulsores: & $\begin{array}{l}\text { Son los sectores que empujan el crecimiento debido al au- } \\
\text { mento en su producción, debido a que requieren más insu- }\end{array}$ \\
\hline Índice de arrastre $>1$. & mos de los demás sectores. \\
\hline Sectores estratégicos: & $\begin{array}{l}\text { Son los sectores con mayor aprovisionamiento de insumos, } \\
\text { por lo que en caso de ocasional incremento en la economía }\end{array}$ \\
\hline Índice de dispersión > 1 . & podrían significar un cuello de botella. \\
\hline Sectores “independientes": Ambos índices < 1 . & $\begin{array}{l}\text { Su integración es escasa con respecto a los demás sectores } \\
\text { del conjunto económico. }\end{array}$ \\
\hline
\end{tabular}

Clasificación de Sectores de la economía de acuerdo con el valor de los Índices de Rasmussen

Fuente: Contabilidad Insumo-Producto y un análisis comparativo-estructural de la economía mexicana (Núñez y Romero, 2016, p. 13). 


\section{Intensidad Energética}

Se denomina intensidad energética a la cantidad de energía utilizada por producción entregada por subsector y uso final. Por lo regular, la intensidad energética se obtiene como la energía consumida dividida por un indicador económico, el cual puede ser el producto interno bruto (PIB) o el valor agregado por sector. Este concepto se establece a través de distintos componentes, entre los cuales se incluyen: eficiencia energética, clase de industria base, tipo de cambio, costo de los servicios energético, dimensión del país, tipo de clima y su comportamiento (IEA, 2015, pp. 17-18) y se aplica como un indicador del crecimiento económico sustentable, además ha sido elegido por la Unión Europea como uno de los catorce indicadores estructurales mediante los cuales se evalúan los avances alcanzados dentro del marco de la Estrategia de Lisboa (Mendiluce, 2010, p. 24).

\section{Eficiencia Energética}

La eficiencia energética, entendida como un recurso energético, tiene la capacidad extraordinaria de coadyuvar a la seguridad energética, al crecimiento económico y también a la mejora de la salud y el bienestar; de manera distintiva constituye un recurso en la disminución de las emisiones de gases de efecto invernadero (IEA, 2015, p. 3). En México se cuenta con el programa denominado "Estadística, Modelación e Indicadores de Eficiencia Energética", y tiene como objetivo la integración y actualización de estadísticas e indicadores de eficiencia energética, a través de los cuales se pueda mejorar el entendimiento de los usos finales de la energía en los diferentes sectores de consumo (Conuee, 2018, pp. 2-3).

Se puede definir como indicador de eficiencia energética al conjunto de acciones establecidas con la finalidad de reducir, con viabilidad económica, la cantidad de energía necesaria para cubrir los requerimientos energéticos de los servicios y bienes que solicita la sociedad, garantizando el nivel de calidad adecuado (DOF, 2015). De acuerdo con Mendiluce y Linares (2011, p. 3), el procedimiento apropiado para evaluar la Eficiencia Energética (EE), en términos macroeconómicos, es mediante la Intensidad Energética (IE). Como anteriormente se ha mencionado, este indicador IE determina el enlace entre consumo energético y volumen de la actividad económica y se calcula como el cociente entre el consumo energético y el producto interno bruto (PIB). Por consiguiente, es el inverso de la eficiencia energética (EE), por lo que es indispensable a fin de mejorar la eficiencia energética reducir la intensidad energética.

$$
\begin{aligned}
I E & =\frac{\text { consumo energético }}{P I B} \\
E E & =\frac{P I B}{\text { consumo energético }}
\end{aligned}
$$




\title{
Mitigación
}

La mitigación se define:

\begin{abstract}
"Cambios y reemplazo tecnológicos que reducen el insumo de recursos y las emisiones por unidad de producción. Aunque hay varias políticas sociales, económicas y tecnológicas que reducirían las emisiones, la mitigación, referida al cambio climático, es la aplicación de políticas destinadas a reducir las emisiones de gases de efecto invernadero y a potenciar los sumideros" (IPCC, 2008, p. 84)
\end{abstract}

Aunque las emisiones de carbono per cápita en México no son altas, la intensidad en el empleo de energía y de carbono es alta en relación con otros países de la OCDE. Por lo tanto, para que México tenga las condiciones necesarios a fin de lograr su objetivo en la disminución de emisiones de gases de efecto invernadero, será primordial implantar precios apropiados, suprimir subvenciones improductivas, al igual que optimizar la eficiencia energética del país (OCDE, 2015, p. 19).

\section{Propuesta Metodológica}

\section{Base de datos}

Para la realización del presente trabajo se utiliza la más reciente matriz de Insumo-Producto de México publicada por el INEGI en 2017, específicamente la matriz simétrica doméstica producto a producto, economía total, origen doméstico, a precios básicos de 2013 para 79 subsectores (INEGI, 2017).

\section{Obtención de los sectores clave en el consumo de energía eléctrica}

Para ilustrar la metodología aplicada en la obtención de los subsectores claves en el consumo de energía eléctrica, aplicaremos un ejemplo simplista de cálculo utilizando la desagregación más detallada disponible y sin entrar en el detalle de las diversas tarifas de precios por tipo de sector y cantidad de consumo, es decir que se considera el consumo de energía eléctrica de tres sectores el cual guarda una relación directa con la tarifa de precios y cantidad de consumo, tal como se muestra en la Tabla 3, considerando la información concerniente a la matriz simétrica de Insumo-Producto, producto a producto, economía total, origen doméstico e importado, a precios básicos de 2013 para 19 subsectores, publicada por el INEGI (2017); 
como nos interesa la fila correspondiente al consumo de energía eléctrica agregada a tres sectores colocamos estos valores y excluimos el renglón del PIB.

Tabla 3

MIP 2013 agregada a tres sectores simplificada, doméstica por tipo de actividad incluyendo consumo de energía eléctrica, precios en Millones de pesos a precios básicos de 2013

\begin{tabular}{cccccc}
\hline Actividades & Sector Primario & $\begin{array}{c}\text { Sector } \\
\text { Industrial }\end{array}$ & $\begin{array}{c}\text { Sector de } \\
\text { Servicios }\end{array}$ & $\begin{array}{c}\text { Demanda } \\
\text { Final }\end{array}$ & $\begin{array}{c}\text { Producción } \\
\text { total }\end{array}$ \\
\hline Sector Primario & 64515 & 421732 & 5170 & 288325 & 779742 \\
Sector Industrial & 117098 & 3021320 & 746758 & 9753926 & 13639102 \\
Sector de Servicios & 55740 & 1853896 & 1805456 & 9508711 & 13223804 \\
$\begin{array}{c}\text { Valor agregado } \\
\text { (542389 }\end{array}$ & 8342154 & 10666420 & 2009673 & 21560636 \\
$\begin{array}{c}\text { Producción total } \\
\text { Consumo de ener- }\end{array}$ & 779742 & 13639102 & 13223804 & 21560636 & 49203284 \\
gía eléctrica & 6404 & 174008 & 101947 & 80209 & 362568 \\
\hline
\end{tabular}

Fuente: Elaboración propia con base en la Matriz Insumo-Producto de México a precios básicos de 2013 (INEGI, 2017).

A partir de estos datos se obtiene mediante un simple análisis que el impacto de la industria eléctrica en la estructura productiva nacional es el siguiente: del total del consumo de energía eléctrica correspondiente a los procesos productivos, el $61.63 \%$ se destina al sector industrial, el $36.11 \%$ al sector de servicios y el $2.27 \%$ al sector primario. Ahora, continuando con nuestro análisis y dado que las unidades de esta matriz Insumo-Producto están indicadas en millones de pesos, aplicaremos el análisis directamente sobre estos datos, teniendo en cuenta que los resultados obtenidos se manejan en porcentajes de cantidades en millones de pesos. La matriz diagonal con el consumo de energía eléctrica está dada por la división del renglón de consumo de energía eléctrica entre la producción total, es decir:

$$
\hat{\boldsymbol{e}}=\left[\begin{array}{lll}
0.01686 & 0.00000 & 0.00000 \\
0.00000 & 0.01443 & 0.00000 \\
0.00000 & 0.00000 & 0.00951
\end{array}\right]
$$


Ahora la matriz inversa de Leontief para tres sectores, está dada por:

$$
\boldsymbol{L}=(\boldsymbol{I}-\boldsymbol{A})^{-\mathbf{1}}=\left[\begin{array}{lll}
1.097702 & 0.044191 & 0.003387 \\
0.220870 & 1.308282 & 0.085661 \\
0.125646 & 0.209605 & 1.171884
\end{array}\right]
$$

Entonces calculando la matriz de multiplicadores de consumo de electricidad, $\widehat{\boldsymbol{e}} \cdot(\boldsymbol{I}-\boldsymbol{A})^{\mathbf{- 1}}$, se tiene:

$$
\widehat{\boldsymbol{e}} \cdot(\boldsymbol{I}-\boldsymbol{A})^{-1}=\left[\begin{array}{lll}
0.009015 & 0.000363 & 0.000028 \\
0.002828 & 0.016691 & 0.001093 \\
0.000969 & 0.001616 & 0.009034
\end{array}\right]
$$

Que corresponde a la matriz de multiplicadores de energía eléctrica (REE), por lo que el impacto total y el índice distributivo se muestran en la Tabla 4, en la cual se presenta la suma de columnas y renglones.

Tabla 4

Efecto total de arrastre y distribución del consumo de Energía eléctrica en los 3 sectores primarios

\begin{tabular}{ccccc}
\hline Actividades & Sector Primario & Sector Industrial & Sector de Servicios & Efecto total de distribución \\
\hline Sector Primario & 0.009015 & 0.000363 & 0.000028 & 0.009406 \\
Sector Industrial & 0.002818 & 0.016691 & 0.001093 & 0.020602 \\
Sector de Servicios & 0.000969 & 0.001616 & 0.009034 & 0.011619 \\
Efecto total de & 0.012802 & 0.018670 & 0.010155 & \\
arrastre & & &
\end{tabular}

Fuente: Elaboración propia con base en la Matriz Insumo-Producto de México a Precios básicos de 2013 (INEGI, 2017).

Ahora, aplicando lo correspondiente a Índices de Rasmussen descrito anteriormente obtenemos los Índices de arrastre y de distribución para el consumo de energía eléctrica para los 3 sectores primarios, información que se presenta en la Tabla 5. 
Tabla 5

Índices de arrastre y distribución del consumo de energía eléctrica en los 3 sectores primarios

\begin{tabular}{cccccc}
\hline \multirow{5}{*}{ Actividades } & \multicolumn{3}{c}{ Matriz de multiplicadores de consumo de } & & \\
& electricidad & & Impacto total \\
& Sector & Sector & Sector de & de dispersión & Índice de distribución \\
& Primario & Industrial & Servicios & & \\
\hline Sector Primario & 0.009015 & 0.000363 & 0.000028 & 0.009406 & 0.677885301 \\
Sector Industrial & 0.002818 & 0.016691 & 0.001093 & 0.020602 & 1.48474654 \\
Sector de Servicios & 0.000969 & 0.001616 & 0.009034 & 0.011619 & 0.837368159 \\
Impacto total arrastre & 0.012802 & 0.018670 & 0.010155 & 0.041627 & \\
& & & Efecto & 0.013876 & \\
& & & medio: & & \\
Índice de arrastre & 0.922612 & 1.345519 & 0.731868 & & \\
\hline
\end{tabular}

Fuente: Elaboración propia con base en la Matriz Insumo-Producto de México a Precios básicos de 2013 (INEGI, 2017).

Por lo que, de acuerdo con la Tabla 5, se puede identificar al sector industrial como el sector clave en el consumo de energía eléctrica en México. Ahora con la finalidad de verificar la aplicación de la ecuación (5) incluimos la demanda final en los datos de Insumo-Producto:

$$
R E E_{p g}=\widehat{e} \cdot(I-A)^{-1} \cdot \hat{y}
$$

Obteniéndose lo siguiente:

$$
\boldsymbol{R E} \boldsymbol{E}_{\boldsymbol{p g}}=\left[\begin{array}{lll}
0.009015 & 0.000363 & 0.000028 \\
0.002828 & 0.016691 & 0.001093 \\
0.000969 & 0.001616 & 0.009034
\end{array}\right] *\left[\begin{array}{c}
288,325 \\
9,753,926 \\
9,508,711
\end{array}\right]=\left[\begin{array}{c}
6,404 \\
174,008 \\
101,947
\end{array}\right]
$$

El resultado nos indica que los valores obtenidos de consumo de electricidad concuerdan con los presentados en la Tabla 3 .

Obtención de la intensidad y eficiencia en el consumo de energía eléctrica

Ahora para obtener estos valores, aplicamos las ecuaciones (9) y (10) con lo que obtenemos la Tabla 6, en la cual se presenta el consumo de energía eléctrica y Producto Interno Bruto (PIB) en millones de pesos a precios básicos de 2013. 
Tabla 6

Intensidad y eficiencia en el consumo de energía eléctrica de acuerdo a MIP 2013 agregada a tres sectores, doméstica por tipo de actividad, precios en millones de Pesos a precios básicos de 2013

\begin{tabular}{ccccc}
\hline Actividades & $\begin{array}{c}\text { Consumo de ener- } \\
\text { gía eléctrica }\end{array}$ & PIB & $\begin{array}{c}\text { Intensidad en el } \\
\text { consumo de energía } \\
\text { eléctrica }\end{array}$ & $\begin{array}{c}\text { Eficiencia en el } \\
\text { consumo de energía } \\
\text { eléctrica }\end{array}$ \\
\hline Sector Primario & 6404 & 476794 & 0.0134 & 74.4532 \\
Sector Industrial & 174008 & 5141987 & 0.0338 & 29.5504 \\
Sector de Servicios & 101947 & 10033297 & 0.0102 & 98.4171 \\
\hline
\end{tabular}

Fuente: Elaboración propia con base en la Matriz Insumo-Producto de México a Precios básicos de 2013 (INEGI, 2017).

\section{Resultados}

Obtención de los subsectores claves en el consumo de energía eléctrica para 79 subsectores

Aplicando la metodología propuesta se identificaron 19 subsectores claves en el consumo de energía eléctrica los cuales se presentan en la Tabla 8. De estos 19 subsectores claves 14 corresponden al sector industrial (marcados con negrita en la Tabla 7) y 5 al sector de servicios. Por otra parte solo 7 sectores identificados como los de mayor consumo de energía eléctrica son subsectores claves (indicados con * en la Tabla 7).

Tabla 7

Subsectores claves en el consumo de energía eléctrica

\begin{tabular}{|c|c|c|c|c|}
\hline No. & $\begin{array}{l}\text { CÓDIGO } \\
\text { CLASE } \\
\text { SCIAN }\end{array}$ & SUBSECTOR & Índice de arrastre & $\begin{array}{l}\text { Índice de } \\
\text { distribución }\end{array}$ \\
\hline 1 & $222 *$ & $\begin{array}{l}\text { Suministro de agua y suministro de } \\
\text { gas por ductos }\end{array}$ & 9.734121 & 11.851983 \\
\hline 2 & $327 *$ & $\begin{array}{l}\text { Fabricación de productos a base de } \\
\text { minerales no metálicos }\end{array}$ & 4.877220 & 5.296454 \\
\hline 3 & 313 & $\begin{array}{l}\text { Fabricación de insumos textiles y } \\
\text { acabado de textiles }\end{array}$ & 3.218297 & 3.985156 \\
\hline 4 & $212 *$ & $\begin{array}{l}\text { Minería de minerales metálicos y no } \\
\text { metálicos, excepto petróleo y gas }\end{array}$ & 2.331348 & 3.890318 \\
\hline 5 & $326^{*}$ & Industria del plástico y del hule & 2.211306 & 2.818198 \\
\hline 6 & $331 *$ & Industrias metálicas básicas & 2.185495 & 2.560128 \\
\hline
\end{tabular}


J. M. E. Vaca Serrano y A. Kido Cruz / Contaduría y Administración 66(2) 2021, 1-22

http://dx.doi.org/10.22201/fca.24488410e.2021.2487

\begin{tabular}{|c|c|c|c|c|}
\hline 7 & $332 *$ & Fabricación de productos metálicos & 2.121818 & 2.164235 \\
\hline 8 & 322 & Industria del papel & 1.684613 & 2.054574 \\
\hline 9 & 321 & Industria de la madera & 1.635226 & 1.717021 \\
\hline 10 & 323 & Impresión e industrias conexas & 1.870621 & 1.518164 \\
\hline 11 & 721 & Servicios de alojamiento temporal & 1.288929 & 1.389916 \\
\hline 12 & $311^{*}$ & Industria alimentaria & 1.104060 & 1.277370 \\
\hline 13 & 339 & Otras industrias manufactureras & 1.318026 & 1.249550 \\
\hline 14 & 512 & $\begin{array}{l}\text { Industria fílmica y del video, e in- } \\
\text { dustria del sonido }\end{array}$ & 1.311059 & 1.219548 \\
\hline 15 & 337 & $\begin{array}{l}\text { Fabricación de muebles, colchones } \\
\qquad \text { y persianas }\end{array}$ & 1.772252 & 1.174112 \\
\hline 16 & 314 & $\begin{array}{l}\text { Fabricación de productos textiles, } \\
\text { excepto prendas de vestir }\end{array}$ & 1.631148 & 1.164285 \\
\hline 17 & 813 & Asociaciones y organizaciones & 1.117352 & 1.047678 \\
\hline 18 & 511 & $\begin{array}{c}\text { Edición de periódicos, revistas, } \\
\text { libros, software y otros materiales, } \\
\text { y ... }\end{array}$ & 1.168013 & 1.008165 \\
\hline 19 & 493 & Servicios de almacenamiento & 1.185662 & 1.007305 \\
\hline
\end{tabular}

Fuente: Elaboración propia con base en la Matriz Insumo-Producto de México a precios básicos de 2013 (INEGI, 2017).

Obtención de la intensidad y eficiencia en el consumo de energía eléctrica

Al igual que para el ejemplo de 3 sectores, aplicamos la ecuación (9) para obtener la intensidad en el consumo de energía eléctrica, obteniéndose los principales subsectores mostrados en la tabla 8.

Tabla 8

Subsectores de mayor intensidad en el consumo de energía eléctrica

\begin{tabular}{cccc}
\hline No. & $\begin{array}{c}\text { CÓDIGO } \\
\text { CLASE } \\
\text { SCIAN }\end{array}$ & SUBSECTOR & $\begin{array}{c}\text { Intensidad en el } \\
\text { consumo de energía } \\
\text { eléctrica }\end{array}$ \\
\hline 1 & 222 & Suministro de agua y suministro de gas por ductos al \\
consumidor final & 0.3298228 \\
2 & 327 & Fabricación de productos a base de minerales no me- & 0.2731411 \\
3 & 313 & Fálicos & 0.1692285
\end{tabular}




\begin{tabular}{cccc}
4 & 326 & Industria del plástico y del hule & 0.1532228 \\
5 & 332 & Fabricación de productos metálicos & 0.1096351 \\
6 & 323 & Impresión e industrias conexas & 0.0908286 \\
7 & 322 & Industria del papel & 0.0902579 \\
8 & 331 & Industrias metálicas básicas & 0.0855081 \\
9 & 329 & Otras industrias manufactureras & 0.0789066 \\
10 & 335 & Fabricación de accesorios, aparatos eléctricos y equipo & 0.0718251 \\
\hline
\end{tabular}

Fuente: Elaboración propia con base en la Matriz Insumo-Producto de México a precios básicos de 2013 (INEGI, 2017).

De acuerdo con el consumo de energía eléctrica y el Producto Interno Bruto correspondiente a cada subsector, los 5 principales subsectores en intensidad de consumo son: Suministro de agua y suministro de gas por ductos al consumidor final, fabricación de productos a base de minerales no metálicos, fabricación de insumos textiles y acabados textiles, industria del plástico y del hule y fabricación de productos metálicos.

Para obtener los principales subsectores de mayor eficiencia en el consumo de energía eléctrica aplicamos la ecuación (10), información que se presenta en la Tabla 9.

Tabla 9

Subsectores de mayor eficiencia en el consumo de energía eléctrica

\begin{tabular}{cclc}
\hline No. & $\begin{array}{c}\text { CÓDIGO CLASE } \\
\text { SCIAN }\end{array}$ & \multicolumn{1}{c}{ SUBSECTOR } & $\begin{array}{c}\text { Eficiencia en el } \\
\text { consumo de energía } \\
\text { eléctrica }\end{array}$ \\
\hline 1 & 238 & Trabajos especializados para la construcción & 1,167 \\
2 & 237 & Construcción de obras de ingeniería civil & 762 \\
3 & 521 & Banca central & 581 \\
4 & 211 & Extracción de petróleo y gas & 524 \\
5 & 482 & Transporte por ferrocarril & 512 \\
6 & 484 & Autotransporte de carga & 506 \\
7 & 483 & Transporte por agua & 497 \\
8 & 236 & Edificación & 422 \\
9 & 531 & Servicios inmobiliarios & 404 \\
10 & 112 & Cría y explotación de animales & 323 \\
\hline
\end{tabular}

Fuente: Elaboración propia con base en la Matriz Insumo-Producto de México a precios básicos de 2013 (INEGI, 2017). 


\section{Conclusión}

La contribución de este estudio con relación a otros trabajos que abordan ese tema, es que se analiza de manera exclusiva el sector eléctrico, identificando 19 subsectores claves en el consumo de energía eléctrica, considerándose el primer estudio de este tipo en México; de igual forma, se determinan los subsectores de mayor intensidad en el consumo de energía eléctrica. Dado que el consumo final de energía eléctrica está en función de los consumos sectoriales, del contenido energético, de la producción de cada sector y de la generación de capital; los resultados obtenidos permiten determinar el enlace del comportamiento económico sectorial con el consumo y demanda de energía eléctrica, con lo cual se pueden plantear escenarios para mejorar la eficiencia en el consumo de electricidad y la mitigación.

De los 19 subsectores claves identificados en el consumo de energía eléctrica, 14 corresponden al sector industrial y 5 al sector de servicios. De los principales subsectores identificados con mayor intensidad en el consumo de energía eléctrica, 8 son subsectores claves en el consumo de este suministro, por lo que se enlistan a continuación: Suministro de agua y suministro de gas por ductos al consumidor final, fabricación de agua y suministro de gas por ductos al consumidor final, industria del plástico y del hule, fabricación de productos metálicos, impresión e industrias conexas, industrias del papel, industrias metálicas básicas y fabricación de accesorios, aparatos eléctricos y equipo de generación de energía eléctrica.

Dado que los subsectores claves y los de mayor intensidad en el consumo de energía eléctrica tienen presencia en todo el país, requieren supervisión especial -a fin de disminuir su intensidad, incrementar su eficiencia y reducir las emisiones de gases contaminantes-, en tanto se determinan las causas de su alto consumo de electricidad y se promueven e instalan tecnologías limpias para generar energía. A fin de que el programa de eficiencia en el consumo sea factible y pertinente, deberá ser elaborado con la participación del gobierno y de las empresas que constituyen los subsectores claves y de mayor intensidad.

Considerando el inmenso potencial de recursos existentes en el país y que la generación de electricidad cuenta con una diversidad significativa de tecnología para su explotación, sería conveniente contar con un programa "más agresivo" de análisis de incorporación y sustitución de centrales de generación provenientes de energía limpia. En México no existe un organismo rector dedicado exclusivamente a este objetivo, sino que esta actividad se encuentra repartida en diferentes áreas de la Secretaría de Energía (SENER), Comisión Federal de Electricidad (CFE) y algunas universidades del país. Recientemente se le ha dado da mayor importancia a la creación y operación del mercado eléctrico mayorista, siendo más trascendental el estudio de la aplicación de los inmensos recursos naturales que tiene el país en el aprovechamiento de generación de electricidad con energía limpia.

En el año 2017 se cuenta con una capacidad instalada de 434 MW de generación distribuida, 
por lo que sería conveniente realizar un análisis de factibilidad técnico-económica a fin de

estimar el potencial incremento de generación con esta modalidad y otras no contaminantes. En consideración de la gran cantidad de emisiones que emiten las 3 centrales carboeléctricas (23.6 Mt de $\mathrm{CO}_{2}$ ) se debe trabajar en un programa de retiro a corto plazo -dado que el retiro de 1400 MW está programado para el año 2029-, sustituyéndolas por otro tipo de tecnología de generación (de preferencia de energía limpia), debido a que en promedio emiten $773 \mathrm{~kg}$ de $\mathrm{CO}_{2}$ por MWh generado, valor por encima de la media de emisiones, en comparación con $346 \mathrm{~kg}$ de $\mathrm{CO}_{2}$ por MWh generado que emite la tecnología de ciclo combinado, valor menor al media del sector eléctrico.

Dentro del Programa de desarrollo del sector eléctrico (PRODESEN) y la prospectiva del sector eléctrico 2018-2032 se indica el retiro de unidades de generación y el incremento de capacidad de generación con energías limpias, sin que se haya observado un programa de sustitución de centrales convencionales por centrales de energía limpia, es decir no se cuenta con una planeación a futuro que implique sustitución de centrales de mayor emisión de contaminantes por las de energías limpias o de menor emisión de contaminantes.

Un inventario de la localización de los centros productores de mayor intensidad de consumo de energía eléctrico y de los subsectores claves en el consumo de energía eléctrica permitirá la sustitución de las centrales convencionales por centrales de energía limpia o de menor emisión de gases de efecto invernadero; o en su caso, el traslado o incorporación de centros de producción en zonas de mayor capacidad de energías limpias. Proyecto que debe contar con la participación del gobierno y de las empresas que constituyen los principales centros industriales de producción.

\section{Agradecimientos}

Al Consejo Nacional de Ciencia y Tecnología (CONACYT) por su valioso apoyo para realizar esta investigación.

\section{Referencias}

Alcántara, Vicent y Padilla, Emilio. (2002). Nota metodológica sobre la determinación de sectores "clave" en el consumo de energía final: una primera aproximación al caso español. Documento de trabajo wps205cast, Department of Applied Economics at Universitat Autonoma of Barcelona. Disponible en https://dialnet.unirioja. es/ejemplar/127825. Consultado: 19/07/2019.

Arreola-Marroquín, J., \& Ríos-Bolívar, H. (2017). Crecimiento económico, precios y consumo de energía en México. Ensayos Revista de Economía, 36(1), 59-78. http://doi.org/S1286-4579(05)00180-2 [pii] 10.1016/j.micinf.2005.04.017 
Betancourt, Y. (2015). Efectos de la aplicación de un impuesto ambiental al CO2 en México: Análisis mediante un Modelo de Insumo-Producto. Tesis de Maestría, Facultad de Economía, Universidad Veracruzana. Disponible en https://cdigital.uv.mx/handle/123456789/46630. Consultado: 02/04/2019.

Bullard, C., \& Herendeen, R. (1975). The energy cost of goods and services. Energy Policy, 3(4), 268-278. https:// doi.org/10.1016/0301-4215(75)90035-X

Cardenete, A., Fuentes-Saguar, P., \& Polo, C. (2014). Seeking a decomposition of CO2 production emissions in the Andalusian economy, Working Paper No. 10.14, Departamento de Economía, Universidad Pablo de Olavide, Sevilla. Disponible en: https://www.researchgate.net/publication/241764697_A_decomposition_of_CO2_production_emissions_in_the_Andalusian_economy. Consultado: 30/01/2018.

CEPAL. (2018). Informe nacional de monitoreo de la eficiencia energética de México 2018. Ciudad de México. Disponible en https://repositorio.cepal.org/bitstream/handle/11362/43612/1/S1800496_es.pdf. Consultado: 29/10/2018.

Conuee. (2018). Estadísticas, modelación e indicadores de Eficiencia Energética. Comisión Nacional para el Uso Eficiente de la Energía. Disponible en https://www.gob.mx/conuee/acciones-y-programas/estadisticas-modelacion-e-indicadores. Consultado: 29/10/2018.

Comisión Reguladora de Energía (CRE). (2017). Certificados de Energías Limpias CEL. Ciudad de México. Retrieved from https://www.gob.mx/cms/uploads/attachment/file/246668/CELs.pdf. Consultado: 29/10/2018.

DOF. (2015). Ley de Transición Energética. Disponible en http://dof.gob.mx/nota_detalle.php?codigo=5421295\&fecha=24/12/2015. Consultado: 29/09/2017.

Gianella, C., \& Canziani, E. (2014). Exploring sustainable low carbon development pathways: situación general de las estrategias de desarrollo bajo en carbono (LCDS) en Perú. Reporte para FES, Pan para el mundo, WWF, CAN-I y Act. Lima, Perú. Disponible en: http://library.fes.de/pdf-files/iez/10908.pdf. Consultado: 18/02/2019.

Guevara, Z., Córdoba, O., García, E., \& Bouchain, R. (2017). The Status and Evolution of Energy Supply and Use in Mexico Prior to the 2014 Energy Reform: An Input-Output. Economies, 5(10). http://doi.org/10.3390/ economies5010010

Guevara, Z., \& Domingos, T. (2017). The multi-factor energy input-output model. Energy Economics, 61, 261269. http://doi.org/10.1016/j.eneco.2016.11.020

Guevara, Z., Molina-Pérez, E., García, E. X. M., \& Pérez-Cirera, V. (2018). Energy and CO2 emission relationships in the NAFTA trading bloc: a multi-regional multi-factor energy input-output approach. Economic Systems Research, 5314. http://doi.org/10.1080/09535314.2018.1528212

Guevara, Z., Rodrigues, J., \& Domingos, T. (2015). The ultimate energy input-output model. In 23rd International Input Output Conference in Mexico City. México, D.F. Disponible en: https://www.iioa.org/conferences/23rd/ papers/files/2029_20150423041_TheultimateEIOmodel.pdf. Consultado: 24/01/2019

Guevara, Z., Sousa, T., \& Domingos, T. (2016). Insights on Energy Transitions in Mexico from the Analysis of Useful Exergy 1971-2009. Energies, 9(7), 488. http://doi.org/10.3390/en9070488

Hartner, M. (2013). A product orientate view on energy use. Ponencia, Energía-Industria-Empleo: Metodología Input-Output (pp. 105-123). Universidad de Deusto. Donostia-San Sebastián. Disponible en: http://www.deusto-publicaciones.es/deusto/pdfs/orkestra/orkestra36.pdf. Consultado: 22/09/2015.

IEA. (2015). Indicadores de Eficiencia Energética: Bases Esenciales para el Establecimiento de Políticas. Paris, Francia. Disponible en https://www.iea.org/publications/freepublications/publication/EnergyEfficiencyVespagnol_epdf.pdf. Consultado: 30/06/2016

INECC, y PNUD. (2012). Estudio del impacto de medidas y políticas de eficiencia energética en los sectores de consumo, sobre el balance de energía y sobre los escenarios de emisiones de gases de efecto invernadero en el corto y mediano plazo. México, D.F. Disponible en https://www.gob.mx/cms/uploads/attachment/file/110171/ CGCCDBC_2012_eficiencia_energetica_en_los_sectores_de_consumo.pdf. Consultado: 30/06/2016.

INEGI. (2017). INEGI- PIB y Cuentas Nacionales. Disponible en http://www.inegi.org.mx/est/contenidos/proyectos/cn/mip13/default.aspx. Consultado: 15/10/2018. 
IPCC. (2008). Cambio Climático 2007 Informe de Síntesis. Ginebra, Suiza. Disponible en https://www.google. com.mx/search?q=Cambio+Clim\%C3\%A1tico+2007+Informe+de+S\%C3\%ADntesis\&oq=Cambio+Clim\%C3\%A1tico+2007+Informe+de+S\%C3\%ADntesis\&aqs=chrome..69i57j0.4312j0j9\&sourceid=chrome\&ie=UTF-8. Consultado: 03/02/2019.

Liu, Q., Chen, Y., Tian, C., Zheng, X. Q., \& Li, J. F. (2016). Strategic deliberation on development of low-carbon energy system in China. Advances in Climate Change Research, 7(1-2), 26-34. http://doi.org/10.1016/j.accre.2016.04.002

Livas-García, A. (2015). Análisis de insumo-producto de energía y observaciones sobre el desarrollo sustentable, caso mexicano 1970-2010. Ingeniería, Investigación y Tecnología, 16(2), 239-251. http://doi.org/10.1016/j. riit.2015.03.008

Loizou, E., Chatzitheodoridis, F., Michailidis, A., Tsakiri, M., \& Theodossiou, G. (2015). Linkages of the energy sector in the Greek economy: an input-output approach. International Journal of Energy Sector Management, 9(3), 393-411. http://dx.doi.org/10.1108/IJESM-06-2013-0004

Mendiluce, M. (2010). La Intensidad Energética en España: Claves para entender su evolución. Tesis Doctoral, Escuela Técnica Superior de Ingeniería, Universidad Pontifica Comillas de Madrid. Disponible en: https:// dialnet.unirioja.es/servlet/tesis?codigo=105694. Consultado: 03/02/2019.

Mendiluce, M., \& Linares, P. (2011). Análisis de la evolución de la intensidad energética en España. economics for energy. Reporte para Economics for Energy. Vigo, España. Disponible en http://www.energiaysociedad.es/ analisis-evolucion-intensidad-energetica-espana/. Consultado: 03/03/2019.

Miller, R., \& Blair, P. (2009). Input-Output Analysis Foundations and Extensions. (Cambrige, Ed.)Cambrigde (Second Edition). Cambrigde. http://doi.org/10.1007/s13398-014-0173-7.2

Núñez, G., \& Romero, J. (2016). Contabilidad insumo-producto y un análisis comparativo-estructural de la economía mexicana * Documento de trabajo No. V-201, Centro de Estudios Económicos, El Colegio de México. Disponible en: https://ideas.repec.org/p/emx/ceedoc/2016-05.html. Consultado: 24/05/2017.

OCDE. (2015). Estudios económicos de la OCDE MÉXICO. Disponible en http://www.oecd.org/economy/surveys/ Mexico-Overview-2015\%20Spanish.pdf. Consultado: 24/05/2017.

ONU. (2019). Cambio Climático. Disponible en http://www.un.org/es/sections/issues-depth/climate-hange/index. html. Consultado: 05/12/2019.

Parra, J. C., \& Pino, O. (2008). Obtención de una matriz insumo-producto a 20 sectores y análisis de los encadenamientos productivos para la región del Bío-Bío, base 2003. Horizontes Empresariales, 7(1), 9-25. http://www. ubiobio.cl/miweb/webfile/media/42/version 7-1/economia.pdf. consultado: 23/04/2017.

Patiño, L. I. (2016). Estructura productiva, eficiencia energética y emisiones de CO2 en Colombia. Tesis Doctoral, Departamento de Economía Aplicada, Universitat Autonòma de Barcelona. Disponible en: https://www.tesisenred.net/handle/10803/400393. Consultado: 17/10/2018.

Ruiz, P. (2014a). Crecimiento bajo en carbono y adopción de tecnologías para la mitigación. Los casos de la Argentina y el Brasil. Reporte para CEPAL, EUROCLOMA y COMISIÓN EUROPEA, Santiago de Chile. Disponible en: https://www.cepal.org/es/publicaciones/36800-crecimiento-carbono-adopcion-tecnologias-la-mitigacion-casos-la-argentina-brasil. Consultado: 03/02/2019.

Ruiz, P. (2014b). Políticas de mitigación del cambio climático en México : un análisis de insumo-producto. Revista Internacional de Estadística y Geografía, 5(1), 16-31. Disponible en: https://rde.inegi.org.mx/index. php/2014/01/09/politicas-de-mitigacion-del-cambio-climatico-en-mexico-un-analisis-de-insumo-producto/. Consultado: 23/11/2018.

SENER. (2018a). PROSEDEN 2018-2032. México, D.F. disponible en https://www.gob.mx/cms/uploads/attachment/file/331770/PRODESEN-2018-2032-definitiva.pdf. Consultado: 03/02/2019.

SENER. (2018b). Prospectiva del Sector Electrico 2018-2032. México, D.F. disponible en http://www.sener.gob. mx/res/PE_y_DT/pub/prospectiva_elect.pdf. Consultado: 03/02/2019. 
SENER, C. (2016). Determinación de la línea base de consumo energético y potenciales de eficiencia energética sectoriales en México. Disponible en: https://www.isi.fraunhofer.de/content/dam/isi/dokumente/ccx/2016/ Linea_Base_de_Consumo_Energetico_en_Marco_Politico_Largo_Plazo_Final_01-06-17.pdf. Consultado: 03/02/2019.

Universidad de Cambridge, \& Consejo Mundial de Energía. (2014). Cambio Climático : Implicaciones para el Sector Energético. Cambrigde. Disponible en https://www.worldenergy.org/wp-content/uploads/2014/06/Publicacion-Cambio-Climatico-implicaciones-para-el-sector-energetico-IPCCC-AR5.pdf. Consultado: 05/02/2019. 